\title{
Hepatic damage associated with dengue-2 virus replication in liver cells of BALB/c mice
}

\author{
Marciano Viana Paes ${ }^{1}$, Henrique Leonel Lenzi ${ }^{2}$, Ana Cristina Martins Nogueira ${ }^{3}$, Gerard James Nuovo ${ }^{4}$, \\ Ângela Teixeira Pinhão ${ }^{5}$, Ester Maria Mota ${ }^{2}$, Carlos Alberto Basílio-de-Oliveira ${ }^{6}$, Hermann Schatzmayr ${ }^{7}$, \\ Ortrud Monika Barth ${ }^{7}$ and Ada Maria de Barcelos Alves ${ }^{1}$
}

One difficulty in studying dengue virus (DENV) is the lack of an experimental model that reproduces the human disease. In a previous work, we have shown that BALB/c mice intraperitoneally inoculated with a DENV-2 isolate presented viremia and mild focal areas of liver injuries. In this study, mice were inoculated by the intravenous route and presented extensive damage areas in the liver tissue, which were evaluated by histopathological and ultrastructural analysis. Hepatic injury was noted mainly around the central vein and portal tracts. Damages consist of hepatocyte injury, including steatosis, swelling and necrosis. Further, erythrophagocytosis, intercellular edema and vascular damages were evident, including hemorrhage, which is characteristic of the dengue-induced hepatitis in human liver. Hepatic lesions were already noted 2 days post infection (p.i.), although effects were more extensive after the seventh day p.i. An increase in alanine aminotransferase and aspartate aminotransferase serum levels was detected 7 and 14 days p.i., respectively, and had correlation to hepatic lesions. Alterations caused by the DENV infection were self-limiting, with a remarkable reduction of all liver damages 49 days p.i. Virus antigens were detected in hepatocytes, Kupffer cells and vascular endothelium, suggesting virus replication in these cells. In situ hybridization, using a probe that anneals in the virus negative RNA strand, showed positive reaction in hepatocytes and vascular endothelium cells of infected mice, thus confirming virus replication in such cells. In general, results revealed that this mouse model reproduces some histopathological effects observed in humans and supports previous findings indicating virus replication in the hepatic tissue.

Laboratory Investigation (2009) 89, 1140-1151; doi:10.1038/labinvest.2009.83; published online 31 August 2009

KEYWORDS: dengue virus; histopathology; in situ hybridization; liver damage; mouse model; transaminase

Dengue virus (DENV) belongs to the Flaviviridae family and consists of four antigenically distinct serotypes (DENV1-4), all causing dengue fever (DF) or dengue hemorrhagic fever/ dengue shock syndrome (DHF/DSS). ${ }^{1}$ It is estimated that about 100 million infections occur each year in tropical and subtropical areas around the world, with approximately 500000 cases of DHF/DSS. ${ }^{2,3}$

The DENV genome consists of a positive single-strand RNA molecule of approximately $11 \mathrm{~kb}$ that encodes a polyprotein precursor, which is co- and post-translationally processed into three structural and seven non-structural proteins. During replication, the virus RNA polymerase (the non-structural 5 protein) synthesizes negative-strand RNA molecules that will further be used as templates for positivestrand RNA, which in turn will be incorporated into the new viral particles. ${ }^{1}$

Although molecular aspects of the virus are well described, the pathogenesis of dengue is still poorly understood. Most of the knowledge was obtained in studies of human cases and suggests that the target cells for virus infection are dendritic cells, monocytes, lymphocytes, hepatocytes and vascular endothelial cells. ${ }^{1,3-6}$ Histopathology of tissue specimens obtained from DF and DHF patients postmortem indicates that lymphoid organs and also the liver are implicated with

'Laboratório de Biotecnologia e Fisiologia de Infecções Virais, Instituto Oswaldo Cruz, Fundação Oswaldo Cruz, Rio de Janeiro, Brazil; ${ }^{2}$ Laboratório de Patologia, Instituto Oswaldo Cruz, Fundação Oswaldo Cruz,Rio de Janeiro, Brazil; ${ }^{3}$ Laboratório de Vacinas Virais, Departamento de Imunologia, Instituto Nacional de Controle de Qualidade, Fundação Oswaldo Cruz, Rio de Janeiro, Brazil; ${ }^{4}$ Department of Pathology, Ohio State University Medical Center, Columbus, OH, USA; ${ }^{5}$ Labortatório de Vírus Respiratório e Sarampo, Instituto Oswaldo Cruz, Fundação Oswaldo Cruz, Rio de Janeiro, Brazil; ${ }^{6}$ Hospital Universitário Gaffrée Guinle, Departamento de Anatomia Patológica, Universidade Federal do Estado do Rio de Janeiro, Rio de Janeiro, Brazil and ${ }^{7}$ Laboratório de Ultraestrutura Viral, Instituto Oswaldo Cruz, Fundação Oswaldo Cruz, Rio de Janeiro, Brazil

Correspondence: Dr AM de Barcelos Alves, Avenida Brasil, 4365, Pav. Leônidas Deane, sala 204, Rio de Janeiro, CEP: 21040-900, Brazil.

E-mail: ada@ioc.fiocruz.br

Received 07 January 2009; revised 13 June 2009; accepted 15 June 2009 
DENV replication. ${ }^{3-9}$ Nevertheless, the knowledge obtained from studies in humans alone is insufficient either to clarify the pathogenesis, or to build strategies on the prevention or treatment of dengue disease. Several mouse models have been developed for the studies with dengue; however, none of them were able to mimic the full spectrum of the disease in humans (reviewed in Bente and Rico-Hesse ${ }^{4}$ and Yauch and Shresta ${ }^{10}$ ).

Our group has recently developed an experimental mouse model to study dengue disease, in which $\mathrm{BALB} / \mathrm{c}$ mice were inoculated intraperitoneally with a non-adapted dengue 2 virus (DENV-2) isolated from a human patient. ${ }^{11}$ Results revealed mild liver injuries and viremia in the infected animals. In this work, BALB/c mice were inoculated by the intravenous (i.v.) route with this DENV-2 isolate and we carried out further studies of liver damages, with a detailed analysis and semi-quantification of parenchyma and vascular injuries, assessment of hepatic enzyme serum levels and evaluation of virus replication in the hepatic tissue. The effects of DENV infection described in this paper correlated with the findings reported in human cases of dengue infection and, therefore, showed that this experimental model can be applied in studies of the pathogenesis of the disease. Moreover, detection of the negative strand of viral RNA in the liver cells supports previous suggestions of dengue replication in the hepatic tissue.

\section{MATERIALS AND METHODS Virus}

The DENV-2 used in these experiments, isolated from a patient serum during an outbreak of the virus in the state of Rio de Janeiro in $1995,{ }^{12}$ was kindly provided by the Flaviviruses Laboratory of Fiocruz, Rio de Janeiro, Brazil. The virus, a non-mouse-adapted sample, was propagated in Aedes albopictus mosquito cell line monolayers (C6/36), with L-15 medium (Sigma, St Louis, MO, USA) supplemented with $1 \%$ non-essential amino acids, $10 \%$ tryptose phosphate broth and $10 \%$ fetal bovine serum, and maintained at $28^{\circ} \mathrm{C}$ for 15 days. Virus was identified by indirect immunofluorescence technique, using a type-specific DENV-2 monoclonal (3H5) antibody as described elsewhere, ${ }^{12}$ and further tittered in C6/36 cells by Reed and Muench method (1938).

\section{Animals and Infection}

All experiments with mice were conducted in compliance with Ethic Principles in Animal Experimentation stated in the Brazilian College of Animal Experimentation and approved by the Institute's Animal Use Ethical Committee. Male BALB/c mice, 2-month old, were i.v. inoculated with DENV-2 (10000 median tissue culture infective dose (TCID50)/0.2 ml) and killed 2, 7, 14 and 49 days post infection (p.i.). Mice i.v. inoculated only with L-15 medium (mock) were used as negative control. All animals were anaesthetized with a mixture of ketamine-xylazine ${ }^{13}$ before bleeding and/or before being killed. For histopathological and ultrastructural analysis, mice were killed by intracardiac perfusion, whereas for biochemical tests animals were bled by retro-orbital puncture at several time intervals. Serum samples were obtained after centrifugation at $2000 \mathrm{rpm}$ for $5 \mathrm{~min}$ and stored at $-70^{\circ} \mathrm{C}$ for further analyses.

\section{Histopathological and Ultrastructural Analysis}

Intracardiac perfusion with $4 \%$ paraformaldehyde in sodium phosphate buffer $(0.2 \mathrm{M}, \mathrm{pH} 7.4)$ was performed for $30 \mathrm{~min}$ in each mice ( $n=5$ in each time point). For histological analysis, the liver of these animals was collected and fragments were dehydrated in ethanol and paraffin-embedded. Liver sections ( $5 \mu \mathrm{m}$ thick) were then treated with different stains (hematoxylin-eosin, Lennert's Giemsa, Masson's trichrome or PAS). These sections were evaluated using a scale ranging from 0 to $4(0=$ none, $1=$ mild, $2=$ moderate, $3=$ severe and focally, and $4=$ severe and diffuse damage), according to the degree of steatosis, hepatocyte swelling, necrosis, intercellular edema and hemorrhage. A total of 10 lobules for each animal were examined and results were plotted as the media of damage values in each mouse. For the correlation between alanine aminotransferase (ALT) or aspartate aminotransferase (AST) levels and hepatic damages on the 14th day p.i., the sum of values obtained for the parameters listed above for each animal was used, which was plotted against serum enzyme levels.

For electron microscopy analysis, fragments of hepatic tissues were post-fixed with $2 \%$ glutaraldehyde in sodium cacodylate buffer $(0.2 \mathrm{M}, \mathrm{pH} 7.2)$, dehydrated in acetone, post-fixed with $1 \%$ buffered osmium tetroxide, embedded in EPON and polymerized at $60^{\circ} \mathrm{C}$ for 3 days. Semi-thin sections $(0.5 \mu \mathrm{m}$ thick) were obtained using a diamond knife (Diatome, Biel, Switzerland) adapted to a Reichert-Jung Ultracut E microtome (Markham, Ontario, Canada). Sections were stained with methylene blue and azure II solu$\operatorname{tion}^{14}$ and were observed using a Zeiss Axiophot light microscope (Zeiss, Jena, Germany). Ultrathin sections (60$90 \mathrm{~nm}$ thick) were stained with uranyl acetate and lead citrate, ${ }^{14}$ and were observed in a Zeiss EM-900 transmission electron microscope (Zeiss).

\section{Biochemical Analysis of Serum Hepatic Enzymes}

Animals $(n=10)$ were bled before or 2, 7 and 14 days after virus inoculation, and serum samples were obtained as described above. Levels of ALT and AST serum enzymes were measured in the biochemical analyzer Reflotron Plus (Roche, Basel, Switzerland).

\section{Immunoperoxidase Technique for the Detection of Viral Antigens}

Paraffin-embedded sections of infected or control animal livers were deparaffinized with three washes in xylol and serial washes in 100, 90 and 70\% ethanol followed by a final wash in PBS. For detection of DENV-2 antigens, samples 
collected 2 and 7 days p.i. were incubated overnight at $4{ }^{\circ} \mathrm{C}$ with an anti-DENV-2 serum raised in monkey (gently provided by Dr R Galler, BioManguinhos, Fiocruz, Rio de Janeiro, Brazil). After washing with Tris- $\mathrm{HCl}(0.05 \mathrm{M}, \mathrm{pH}$ 7.6), sections were further incubated with rabbit anti-human IgGhorseradish peroxidase conjugate (Sigma) for $30 \mathrm{~min}$ at $37^{\circ} \mathrm{C}$. Slices were revealed with DAB (Sigma) and counterstained with Mayer's hematoxylin. One control sample (mock) was stained as described above and another control (liver of a 7-day infected mouse) was stained only with the secondary antibody. The percentage of positive cells was determined in three liver lobules of each animal.

\section{In Situ Hybridization}

For in situ hybridization, we used one probe with the sequence $5^{\prime}$-TGACCATCATGGACCTCCA- $3^{\prime}$ that anneals in a conserved region inside the NS3 gene in the negative strand of viral RNA, and contained six dispersed locked nucleic acid modified bases with digoxigenin conjugated to the $5^{\prime}$ end. Paraffin-embedded sections of infected or control animal livers $(5 \mu \mathrm{m})$ were treated for in situ hybridization as described elsewhere. ${ }^{15}$ Briefly, deparaffinized sections were digested with pepsin $(1.3 \mathrm{mg} / \mathrm{ml})$ for $30 \mathrm{~min}$, incubated with the probe cocktail at $60^{\circ} \mathrm{C}$ for $5 \mathrm{~min}$ for denaturation, followed by hybridization at $37^{\circ} \mathrm{C}$ overnight. Sections were further washed with $0.2 \times$ SSC and $2 \%$ bovine serum albumin at $4{ }^{\circ} \mathrm{C}$ for $10 \mathrm{~min}$, and the probe-target complex was visualized due to the action of alkaline phosphatase on the chromogen nitroblue tetrazolium and bromochloroindolyl phosphate.

\section{Statistical Analyses}

Statistical analyses were performed using GraphPad Prism software (La Jolla, CA, USA), version 4.03. For histopathological alterations, Mann-Whitney test was used and values were considered significant at $P<0.05$. Correlations between histopathological damages and transaminase levels were analyzed by linear regression.

\section{RESULTS}

\section{Liver of Infected Mice: Histopathological Aspects}

All mice i.v. inoculated with DENV-2 survived the infection and did not present apparent clinical signs, although damage was observed in the liver of these animals. As expected, liver of non-infected mice showed regular structured hepatic parenchyma with hepatocytes around the central veins and sinusoid capillaries exhibiting normal endothelial cells (Figure 1a). On the second day p.i., histopathological analysis revealed liver tissue injury evidenced by steatosis (micro and macro), hepatocyte swelling, intercellular edema, hemorrhage and necrosis with an inflammatory infiltrate that included mostly neutrophils (Figures $1 \mathrm{~b}$ and c). Engulfing of erythrocytes close to the nucleus of mononucleated and binucleated swelled hepatocytes (erythrophagocytosis) was also noted (Figure 1d). On the seventh day p.i., damages were intensified, affecting more extensive areas that presented mainly macrosteatosis, binucleated swelled hepatocytes and necrosis with an increase of erythrophagocytosis (Figures 1e-g). On the 14th day p.i., injury was still more evident, with extensive areas of inter- and intracellular edema located mainly in midzonal areas, and disruption of endothelium in several central veins (Figure 1h). Lymphocyte infiltrates were also observed in contact with swelled hepatocytes (Figure 1h), whereas necrosis was detected in sinusoid capillaries with an increase of polymorphonuclear cells (Figure 1i). On the other hand, on the 49th day p.i., damages were remarkably reduced and hepatic parenchyma was more similar to that observed in non-infected mice. Alterations were observed mainly due to hepatocyte regeneration, as revealed by the presence of binucleated hepatocytes (Figure 1j). Nevertheless, the presence of erythrophagocytosis, voluminous hepatocytes with complete eosinophilic granulations, which is suggestive of large mitochondria and mononuclear infiltrates could still be detected in some areas (Figures $1 \mathrm{k}$ and $\mathrm{l}$ ).

The histopathological alterations were then semi-quantitatively evaluated at each time point. Hepatocyte injuries as steatosis and intracellular edema were detected from the 2nd day p.i., although peak damages were statistically significant only after the 7 th day and remained similar until the 14th day p.i. (Figure 2a). Necrotic hepatocytes were also detected on the second day p.i., whereas it was statistically significant only on the seventh day p.i. and decreased during the following days (Figure 2b). On the other hand, the kinetic of vascular alterations showed distinct patterns. Peak of intercellular edema was statistically significant only on the 14th day p.i., whereas hemorrhage was present mainly in the beginning of the infection (Figure 2c). In contrast, on the 49th day p.i, all liver injuries presented a dramatic decrease, reaching levels similar to those founded in control mice.

\section{Ultrastructural Aspects of Infected Liver}

Before the evaluation of hepatic cells by electron microscopy, livers of DENV infected mice were also observed by semithin analysis, confirming the observations made in paraffin sections. On the second day p.i., hepatocytes presented large lipid droplet inclusions (macrovesicular steatosis) (Figure 3b), which were absent in control mice (Figure 3a). On the seventh day p.i., numerous inflammatory cells (mainly lymphocytes and Kupffer cells), and hyperplasic plates of swollen and binucleated hepatocytes could be detected in several focal areas (Figure 3c). These effects were even more exacerbated on the 14th day p.i., in which swelling and macrovesicular steatosis were more diffuse (Figure 3d). At this time point of the kinetic of infection, several dilated sinusoid capillaries could be detected, which leads to fluid extravasations (edema/plasma leakage) (Figure 3d). In contrast, on the 49th day p.i., sinusoid capillary dilatations were absent and regeneration of the tissue was also suggested by the presence of binucleated hepatocytes (Figure 3e). However, some isolated hepatocytes presented prominent 

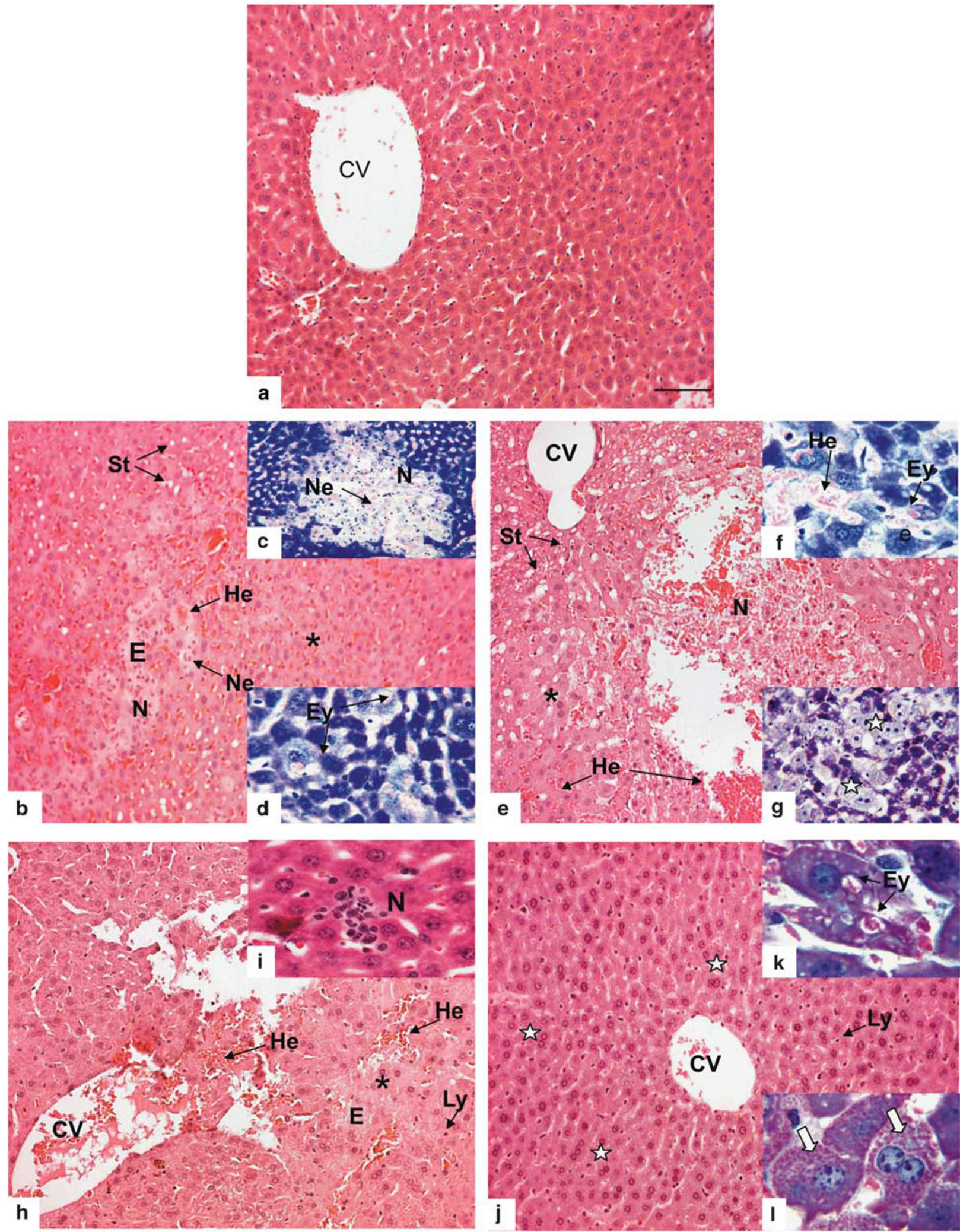

Figure 1 Histopathology analysis of liver sections of control and DENV-2-infected mice. (a) Liver of non-infected mice, showing normal aspect (H\&E, bar $=90 \mu \mathrm{m}$ ). (b-d) Liver section of a mouse killed 2 days p.i. (b, H\&E, bar $=90 \mu \mathrm{m}$; c and d, Lennert's Giemsa, bar $=200 \mu \mathrm{m}$ and bar $=100 \mu \mathrm{m}$, respectively). (e-g) Liver section collected 7 days p.i. (e, H\&E, bar $=90 \mu \mathrm{m} ; \mathbf{f}$, Lennert's Giemsa, bar $=70 \mu \mathrm{m} ; \mathbf{g}$, P.A.S., bar $=100 \mu \mathrm{m})$. (h and i) Liver section obtained 14 days p.i. (H\&E, bar $=90 \mu \mathrm{m}$ and bar $=70 \mu \mathrm{m}$, respectively). (j-I) Liver section observed 49 days p.i. (j, H\&E, bar $=90 \mu \mathrm{m} ; \mathbf{k}$ and $\mathbf{I}$, Masson's trichrome, bar $=40 \mu \mathrm{m})$. CV, central vein; ST, steatosis; He, hemorrhage; E, intercellular edema; N, necrosis; $\mathrm{Ne}$, neutrophil; ${ }^{*}$, hepatocyte swelling; Ey, erythrophagocytosis; stars, binucleated hepatocytes; Ly, lymphocyte; white arrows, eosinophilic granulation in hepatocytes. 

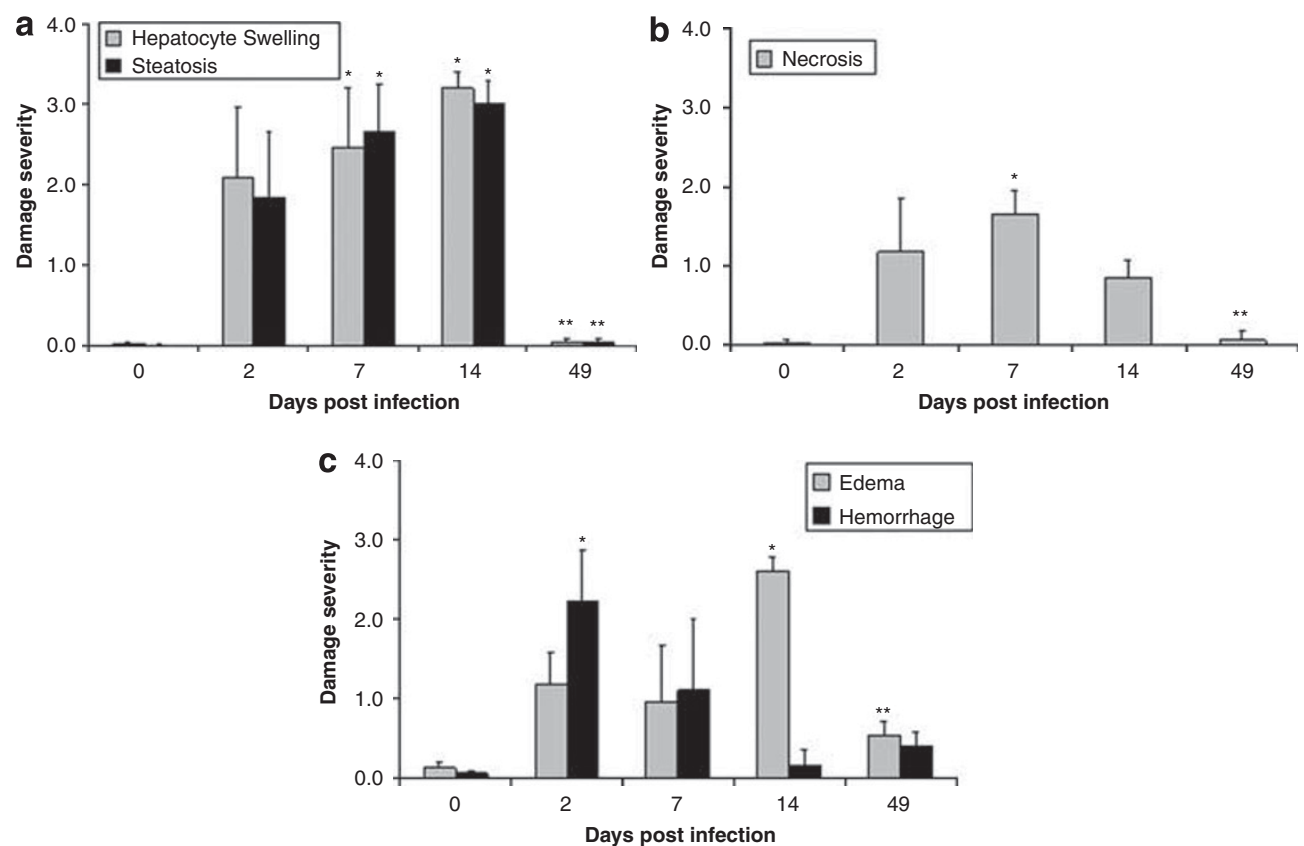

Figure 2 Semi-quantitative analysis of hepatic damage in DENV-2-infected mice observed in several time points, using a subjective scale ranging from 0 to $4(0=$ none, $1=$ mild and focally, $2=$ moderate and focally, $3=$ severe and focally and $4=$ severe and diffuse damage), according to injury degree. A total of 10 lobules for each animal were examined and results were plotted as the media of damage values in each mouse. Damage quantifications were based on steatosis and hepatocyte swelling (a), necrosis (b), hemorrhage and intercellular edema (c). Asterisks indicate differences that are statistically significant between negative control and infected mice $\left.{ }^{*}\right)$ or between peak damage and 49th day p.i. $\left(^{* *}\right)(P<0.05)$.

mitochondria profile, accrediting a granular aspect of the cytoplasm.

A detailed analysis of the alterations caused by DENV infection was performed by electron microscopy. As expected, non-infected liver showed very well-structured sinusoid capillary and hepatocytes (Figure 4a). In contrast, on the second day p.i., hepatocytes frequently showed large lipid inclusions (Figure $4 \mathrm{~b}$ ), which were strongly marked on the seventh day p.i., with numerous of pleomorphic lysosomes accompanied by small and large lipid inclusions (Figure 4c). Platelets, monocytes and lymphocytes appeared in the lumen of sinusoids in closer proximity to hepatocytes, with concomitant endothelium denudations (Figures $4 \mathrm{c}$ and $\mathrm{d}$ ). Endothelium destructions were still more pronounced on the 14th day p.i. (Figure 4e). On the 49th day p.i., most hepatocytes presented mitochondria with normal aspects (Figure 5a), although some cells in focal areas exhibited changes in these organelles, expressed mainly by the swelling of its matricial chamber (Figure 5b). At this time point, an increase of infiltrated cells inside the sinusoidal lumen, such as platelets, lymphocytes, large monocytes (macrophage-like) and cell debris, was also observed.

\section{Quantifications of Serum Transaminases Levels in DENV-2 Infected Mice}

To investigate whether the hepatic damages caused by DENV infection correlate to the changes in transaminase levels, quantitative analyses of ALT and AST were performed. Levels of both the serum enzymes significantly increased after virus infection, showing peak detection on the 7th day p.i. for ALT (Figure 6a) and on the 14th day p.i. for AST (Figure 6b). Moreover, to confirm whether the increase in the serum levels of ALT and AST was due to injuries observed in mouse livers, we tested the correlation between these enzymes and hepatic damages. Regression curves showed 65 and $70 \%$ of correlation between hepatic damages and ALT and AST levels, respectively (Figures $6 \mathrm{c}$ and d). These results underlined that the increase in transaminase levels observed is possibly due to the cytotoxic effects of infection in the liver.

\section{Detection of Viral Antigens in Liver of Infected Mice}

The presence of DENV-2 in the liver tissue of infected mice was investigated by immunohistochemistry assays, using antiDENV-2 polyclonal antibodies raised in monkeys. As expected, negative control, constituted of liver of mockinoculated mice reacting with anti-DENV-2 antibodies or the liver of infected mice reacting with anti-human IgG conjugate, did not present any positive reaction (Figures $7 \mathrm{a}$ and $b)$. On the other hand, DENV-2 antigens could be observed in hyperplasic Kupffer cells in sinusoid capillaries, or in hepatocytes and capillary endothelial cells around portal space or central veins of infected animals (Figures $7 \mathrm{c}-\mathrm{e}$ ). Quantification of positive cells indicated that 2 and $4 \%$ of hepatocytes expressed virus antigens on the second and seventh days p.i., respectively, whereas the presence of positive Kupffer cells ranged from 17 to $12 \%$ at these time points of infection. Results suggested that the DENV-2 replicates in such cells, which are present in the same areas of the observed damages. 

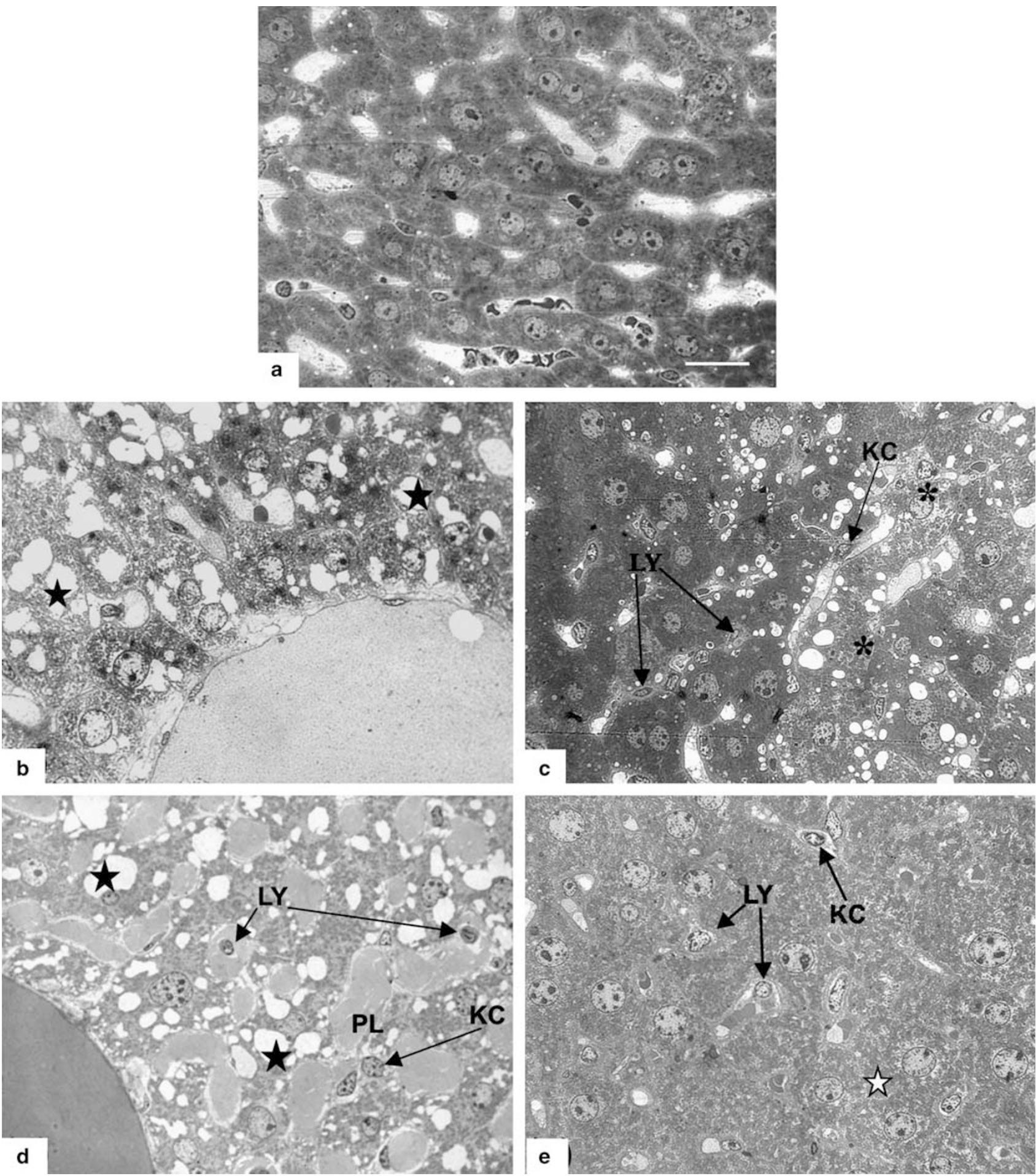

Figure 3 Liver semi-thin sections of control and DENV-2-infected mice, stained with methylene blue and azure II solution. (a) Liver parenchyma from noninfected mice with normal aspect (bar $=2 \mu \mathrm{m}$ ). (b) Liver of one mouse killed 2 days p.i. showing hepatocytes with large lipid droplets (black stars) in the periportal area $(\mathrm{bar}=6 \mu \mathrm{m}$ ). (c) Damages observed 7 days p.i. presenting heterogeneous parenchyma, which consists of dark and compact hepatocytes flanked by clear and swollen (asterisks) or regenerated cells (bar $=3 \mu \mathrm{m})$. (d) Sections obtained 14 days p.i. showing more extensive areas of large lipid droplets (black stars) and plasma leakage (PL). (e) Section observed 49 days p.i. presenting binucleated hepatocytes with speckled cytoplasm (white star) $(\mathrm{bar}=2 \mu \mathrm{m})$. Ly, Lymphocytes, KC, Kupffer cells.

Detection of Virus Replication in Liver Tissue of Infected Mice Replication of the DENV-2 in the liver tissue of infected mice was confirmed by in situ hybridization using a probe that anneals in the negative strand of the viral RNA. Results showed the presence of virus negative strand RNAs in hepatocytes and vascular endothelium cells in mouse liver 

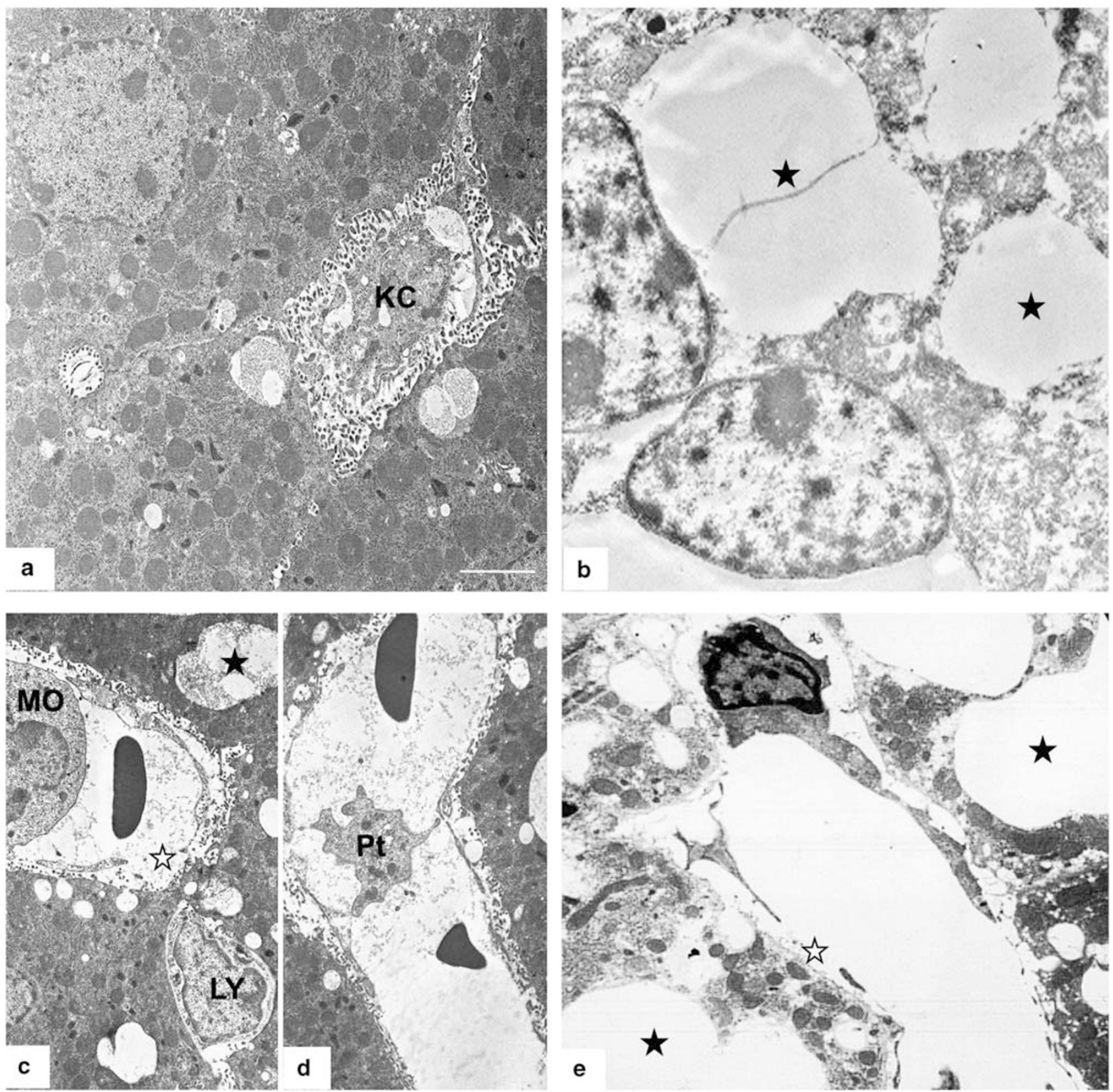

Figure 4 Ultrathin sections of control and DENV-2-infected mouse livers, stained with uranyl acetate and lead citrate. (a) Liver of a non-infected mouse presenting normal hepatocytes and regular sinusoidal border with short finger-like cytoplasmic extensions (bar $=1.4 \mu \mathrm{m}$ ). (b) Liver section of a mouse killed 2 days p.i. showing large lipid inclusions in the cytoplasm of hepatocytes (black stars) (bar $=1.4 \mu \mathrm{m})$. (c and d) Liver sections collected 7 days p.i. showing pleomorphic lysosomes containing flocculent material in the hepatocyte (black star), the presence of platelet (Pt), monocyte (MO) and lymphocyte (LY) inside sinusoids with endothelium denudation (white star) (bars $=1.6$ and $1.5 \mu \mathrm{m}$, respectively). (e) Liver section obtained 14 days p.i., in which endothelium denudation (white star) and macrovesicular steatosis (black stars) were still more pronounced (bar $=1.5 \mu \mathrm{m}$ ).

tissues obtained 7 days p.i. (Figure 8b). As expected, no reaction was observed in tissue from mock-inoculated mice (Figure 8a). Such results confirmed those observed by immunohistochemistry assays, thus corroborating virus replications in the mouse liver tissue.

\section{DISCUSSION}

Despite the worldwide importance of dengue disease, the comprehension of the pathophysiology of DF and DHF is limited, as tissues samples can only be obtained from fatal cases. Therefore, the development of experimental animal models is an essential scientific attempt to solve this problem. ${ }^{4,10}$ Although non-human primates are normally chosen for testing vaccines and/or antiviral against flavivirus before clinical trials, this model is prohibitive in terms of cost and accessibility for studying basic questions about these pathogens. On the other hand, several mouse models have been described for dengue studies, although none of them were 

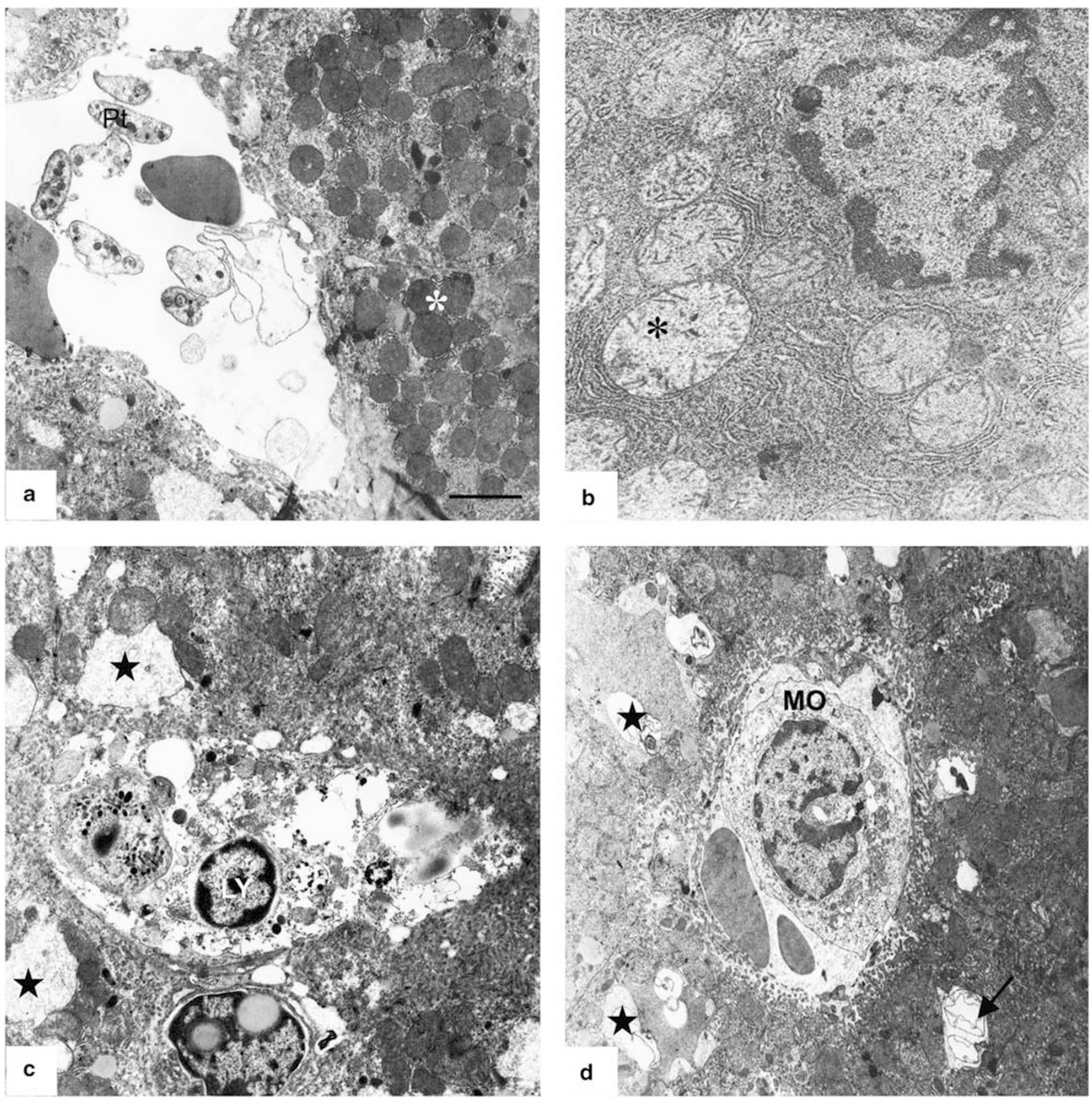

Figure 5 Ultrathin sections of mouse livers obtained 49 days p.i., stained with uranyl acetate and lead citrate. (a and b) Sections presenting mitochondria with normal aspects (white asterisk) in a regenerated hepatocyte (a) and large swollen altered organelles (black asterisk) (b) (bars $=6$ and $1.2 \mu \mathrm{m}$, respectively). (c and d) Sections still showing cell injury presenting pleomorphic lysosomes (black stars), with small membranous whorls in the cytoplasm (arrow), and infiltrated cells inside the sinusoidal lumen (bars $=1.3$ and $1.4 \mu \mathrm{m}$, respectively). Pt, platelet; LY, lymphocyte; MO, monocyte.

able to mimic the full spectrum of the disease., ${ }^{4,10,14,16-25}$ Difficulties are the result of many factors, including the inability of human clinical isolates to replicate well in mice, which frequently are overcome by the use of brain-mouse adapted viruses, non-physiological routes of infection and the use of immunodeficient and/or humanized animals. ${ }^{4,16,17,24,25}$ Although these models are useful for many studies with DENV, they all have limitations, especially because of a loss of some human pathogenic properties that may not reflect natural events. Moreover, manipulated animals, lacking critical components of the host immune system, are not ideal for testing prophylactic vaccines or therapeutical drugs, as responses in these animals are not the same as in wild type, and conclusions about the efficacy and/or potency of medicaments may be compromised.

Investigations of DHF/DSS fatal cases indicated that the liver is one of the most affected organ. ${ }^{7-9,26-28}$ In a previous work, we have shown that BALB/c mice intraperitoneally 

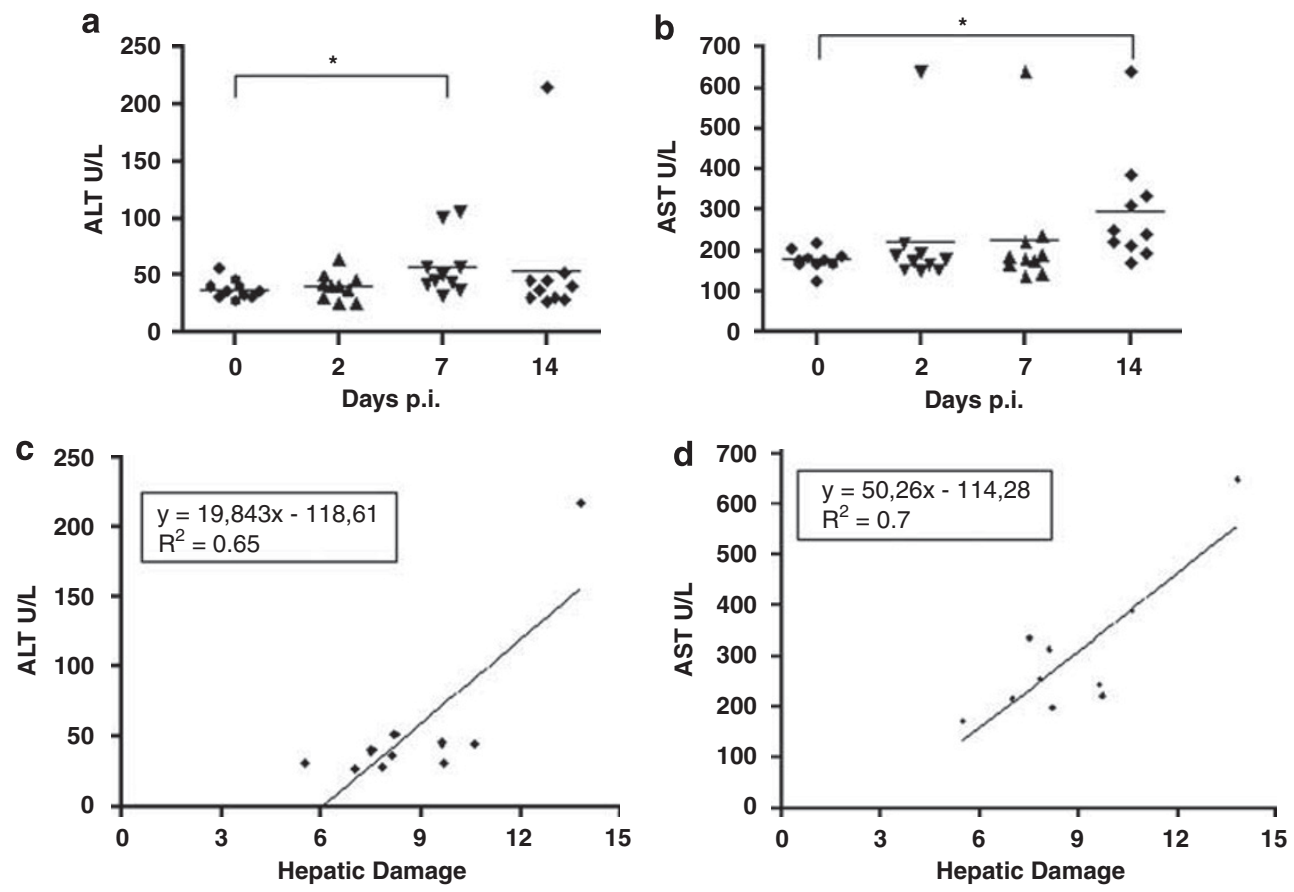

Figure 6 Serum levels of (a) alanine aminotransferase (ALT) and (b) aspartate aminotransferase (AST) in mice experimentally infected with DENV-2, at the 2nd, 7th and 14th day p.i., as illustrated by different symbols. Asterisks indicate differences that are statistically significant $\left({ }^{*} P<0.05\right)$. Correlation tests between ALT (c) or AST (d) levels at the 14th day p.i. and hepatic damages were performed by linear regression. For hepatic damage quantifications, the sum of values obtained for steatosis, hepatocyte swelling, necrosis, hemorrhage and edema for each animal at the 14th day p.i. was calculated and then plotted against serum enzyme levels.

inoculated with a DENV-2, isolated from a human patient, presented viremia and mild focal areas of liver injuries. ${ }^{11}$ In this work, mice were inoculated by the i.v. route with this DENV-2 isolate and we carried out further studies of liver damages, with a detailed analysis and semi-quantification of parenchyma and vascular injuries. Data indicated that the i.v. route led to earlier and more extensive areas of liver damages when compared with our previous work. The most evident tissue alterations observed here were steatosis, hepatocyte swelling, necrosis, areas of hemorrhage and edema/plasma leakage, similar to the effects described in fatal human cases. $^{8,26,27,29}$ All histopathological aspects were mainly observed in zone II (midzonal areas) in the liver hepatic acini, and sinusoidal infiltrate was evident in zones I (periportal areas) and II of the acinus, correlating to the findings described in humans. ${ }^{8,9,27}$ The detection of steatosis in mice was in agreement with other findings, which showed that it is a microscopic feature in the liver of dengue fatal human cases, ${ }^{8}$ normally associated with hepatomegaly. However, although steatosis represents a metabolic disturbance observed in several hepatitis of virus origin such as yellow fever ${ }^{30}$ and hepatitis $C,{ }^{31}$ its pathogenesis in dengue infections is not fully understood. In this study, steatosis was a reversible effect and was not accompanied by fibrosis, thus reflecting a mild effect of dengue infection, which may be associated to DF.

In addition to these alterations, we also observed the engulfing of erythrocytes close to the nucleus of few mononucleated and binucleated swelled hepatocytes, indicating an erythrophagocytosis process. The hemophagocytic syndrome has been reported in some cases of DHF, ${ }^{32-35}$ which might be associated to a wide spectrum of the disease severity, from mild to severe. ${ }^{34}$ The erythrophagocytosis in these human cases were detected mainly in mature macrophage of bone marrow. ${ }^{32-35}$ Thus, as far as we know, results here presented are the first report of the erythrophagocytosis process caused by dengue infection in the liver tissue and involving hepatocytes. However, the exact mechanism and extension of such phenomenon remain to be established.

Besides similarities between our findings and human pathological reports, the mouse model here presented allowed us to track the emergence and development of liver damages, which can in the future contribute toward revealing mechanisms involved in the pathogenesis of the disease. Our semi-quantitative analyses showed that the injuries were already present 2 days after infection, although peak damages were mainly observed around the seventh day p.i. Curiously, vascular damages presented distinct kinetics, in which hemorrhage appeared in the beginning of infection and edema were more pronounced in later stages. The vast majority of liver damages detected after infection with DENV-2 was reversible and at the 49th day p.i. parenchyma regeneration was observed, showing that the infection was self-liming. These data correlates with the hepatic dysfunction observed in patients suffering from DF, wherein initial 

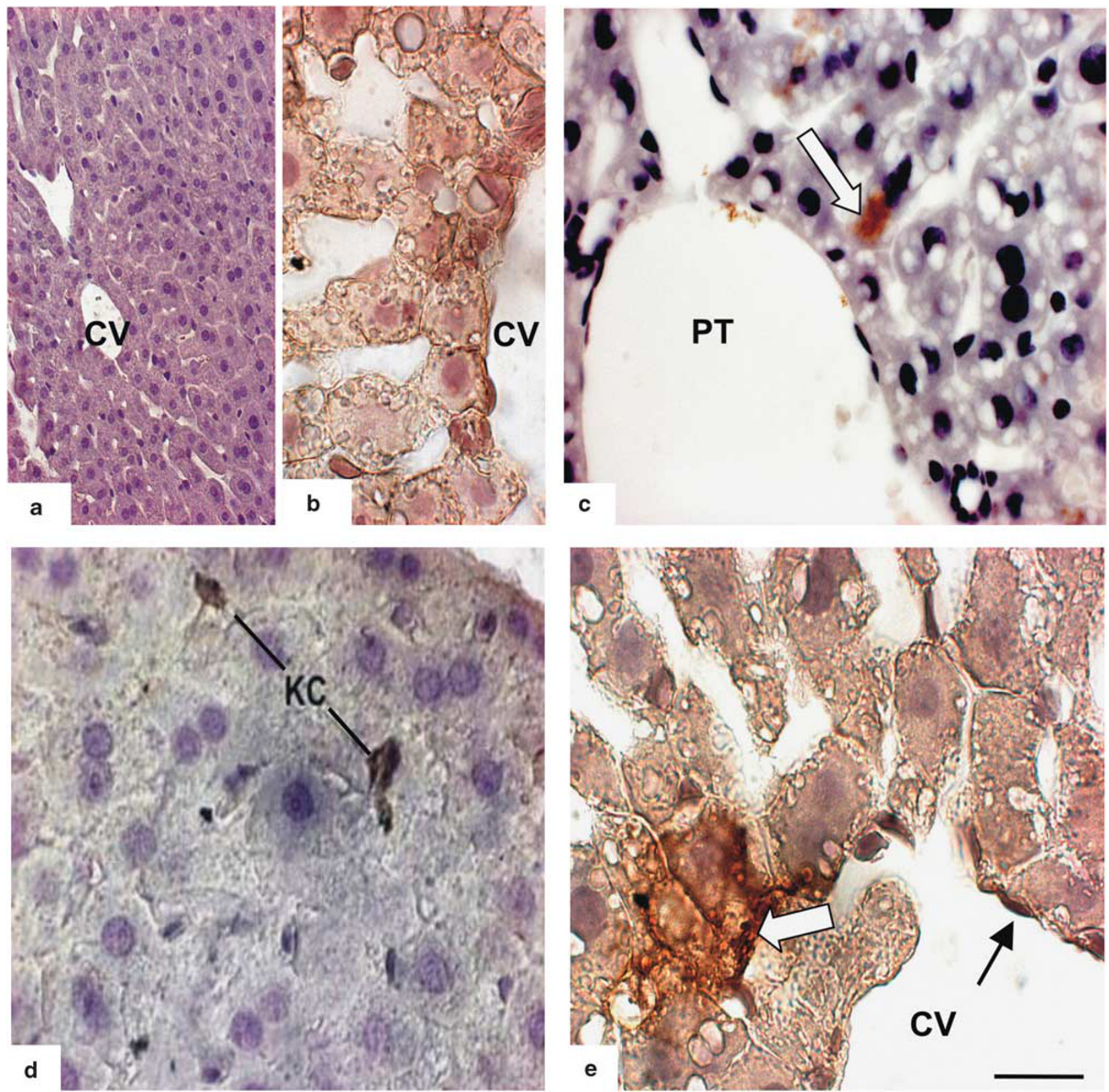

Figure 7 Detection of DENV-2 antigens in liver of infected mice. (a) Negative control of a non-infected mouse liver incubated with anti-DENV-2 and antihuman IgG-horseradish peroxidase conjugate (bar $=2 \mu \mathrm{m}$ ). (b) Liver of a 7-day DENV-2-infected mouse incubated with only anti-human IgG conjugate (bar $=5 \mu \mathrm{m}$ ). (c-e) Mouse livers obtained 2 days (c and d) or 7 days (e) p.i., incubated with anti-DENV-2 and anti-human IgG conjugate (bars $=5,2$ and $3 \mu \mathrm{m}$, respectively). Note focal hepatocytes exhibiting virus antigens around portal space (c) and central vein (e) (white arrows). Virus antigens were also observed in hyperplasic Kupffer cells inside sinusoid capillaries (d) and endothelial cells (e) (black arrow). CV, central vein; PS, portal space; KC, Kupffer cells.

liver failure was reversible. ${ }^{36-39}$ However, very few reports are available concerning histopathological aspects in liver of human non-fatal DF cases, ${ }^{36}$ which might be less severe than in DHF.

Ultrastructural analyses confirmed the results obtained with histopathological data. Mild infiltrates, mainly lymphocytes, platelets and monocytes, were observed in sinusoid capillaries, most of them presenting endothelium denuda- tion, and are in agreement with the data published on human cases of dengue infections. ${ }^{78}$ In fact, dengue, as well as other flavivirus diseases like yellow fever, is characterized by its peculiar pathogenesis, in which an intense hepatic injury is described, which is disproportional to the modest pattern of inflammatory infiltrates observed. ${ }^{7,8,38}$ Damages included several hyperplasic hepatocytes, which exhibited large cytoplasmic lipid droplets, pleomorphic lysosomes and 

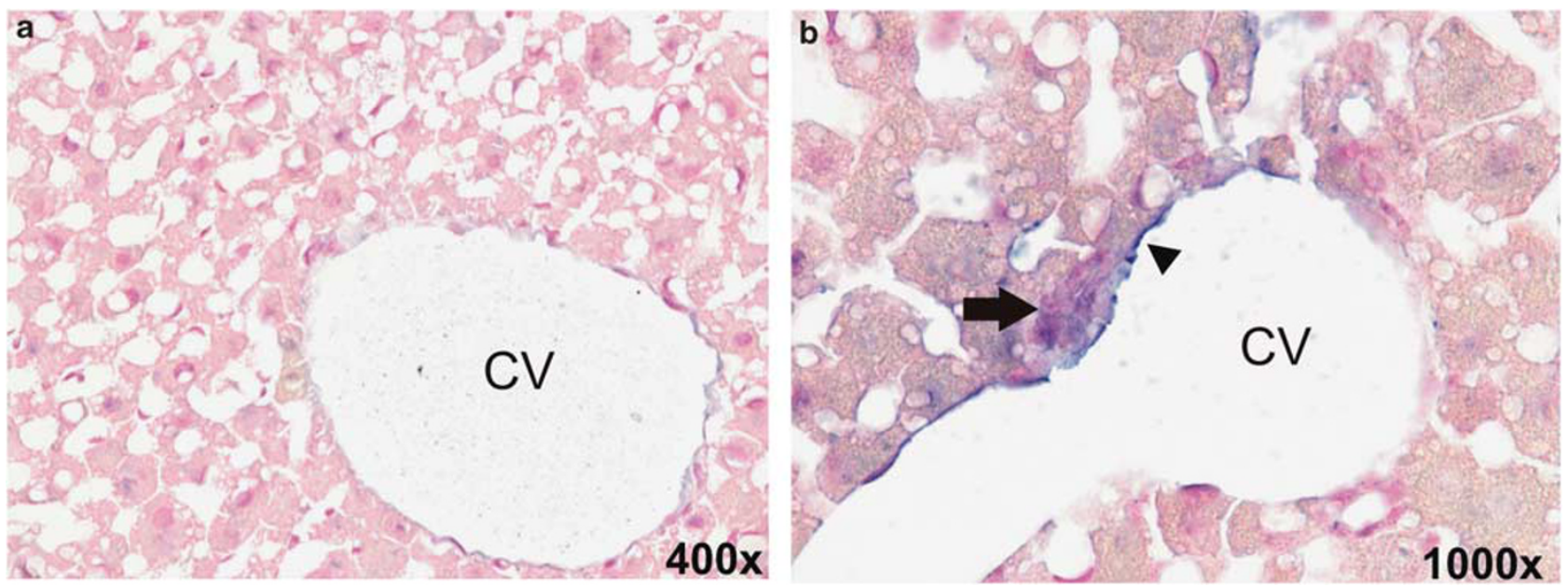

Figure 8 Detection of DENV-2 RNA negative strand by in situ hybridization in liver tissue of infected mice. (a) Negative control of a non-infected mouse; (b) liver of a mouse 7 days p.i. Arrows and arrowhead indicate positive hepatocytes and vascular endothelium cells, respectively. CV, central vein.

mitochondria swelling. The alterations on mitochondria morphology suggest an apoptosis process. Indeed, apoptosis has been described in several studies, both in vivo and in vitro. Liver biopsies obtained from either children or adults infected with DENV presented several apoptotic cells, mainly hepatocytes and Kupffer cells. ${ }^{7,8,37,40,41}$ In vitro infection of a hepatocyte cell line with DENV-2 also showed mitochondria swelling and dysfunction associated with alterations in cellular ATP balance, which is related to the apoptotic process. ${ }^{42}$

We also evaluated hepatic injury by measurements of serum transaminase levels. We observed a significant increase of the enzymes ALT and AST on the 7th and 14th day p.i., respectively. The increase on these enzyme levels, together with thrombocytopenia, is one of the most remarkable features of dengue disease, detected in DF cases and even more in DHF/DSS. ${ }^{36-39,42,43}$ Furthermore, in our mice model a statistical significant correlation between the quantified hepatic injury and serum ALT and AST levels was observed.

Besides liver damages, we also investigated infection and virus replication in the hepatic tissue of DENV-2 i.v. inoculated mice. Dengue antigens were detected in hepatocytes, either in the vicinity of portal tract or around central veins, Kupffer cells and vascular endothelium, which correlate to what were observed in fatal human cases. ${ }^{7,9,36,44,45}$ These data suggest that DENV replicates in the liver tissue. To confirm such hypothesis, we performed an in situ hybridization using a probe that anneals in a conserved region of the negative strand of viral RNA. In fact, a positive reaction was observed in hepatocytes and endothelium cells around central vein, thus confirming our previous observation and supporting suggestions of dengue replication in the human hepatic tissue. ${ }^{7,8,11}$

In summary, the effects of DENV infection described in this paper correlate to some findings reported in human cases of dengue disease and revealed the ability of a virus circulating in a human population to infect $\mathrm{BALB} / \mathrm{c}$ mice.
Moreover, as the effects of infection were reversible and determined mainly by tissue injuries observed in focal areas, we believe that our model is more closely related to DF than to DHF, and may be a useful tool for the study of the pathogenesis of dengue disease. In addition, this model is also an appropriate physiological approach for testing both vaccines and therapeutics. ${ }^{46}$ Further studies for investigation of the effect of the DENV2 infection in other organs are currently in progress, which might contribute to establish the target tissues and cells involved in the pathogenesis of the disease, as well as the mechanisms behind it.

\section{ACKNOWLEDGEMENTS}

We thank Dr Rita Nogueira (Laboratory of Flaviviruses, Instituto Oswaldo Cruz, Fiocruz) for supplying the dengue 2 isolate and Dr Ricardo Galler (BioManguinhos, Fiocruz) for the anti-DENV-2 serum raised in monkey. We are also grateful for the technical assistance of Aline Vilas Boas Vianna. This work was supported by PDTIS-Fiocruz, CNPq, FAPERJ and Instituto do Milênio-MCT.

\section{DISCLOSURE/CONFLICT OF INTEREST}

The authors declare no conflict of interest.

1. Lindenbach $B D$, Rice $C M$. Flaviviridae: the viruses and theireplication. In: Knipe DM, Howley PM (eds). Fields Virology. Lippincott Williams and Wilkins: Philadelphia, 2001, pp 991-1041.

2. Gubler DJ. Dengue and dengue hemorrhagic fever. Clin Microbiol Rev 1998;11:480-496.

3. Leong AS, Wong KT, Leong TY, et al. The pathology of dengue hemorrhagic fever. Semin Diagn Pathol 2007;24:227-236.

4. Bente DA, Rico-Hesse R. Models of dengue virus infection. Drug Discov Today Dis Models 2006;3:97-103.

5. Hall WC, Crowell TP, Watts DM, et al. Demonstration of yellow fever and dengue antigens in formalin-fixed paraffin-embedded human liver by immunohistochemical analysis. Am J Trop Med Hyg 1991;45:408-417.

6. Rosen L, Drogue MT, Deubel V, et al. Detection of dengue virus RNA by reverse transcription-polymerase chain reaction in the liver and lymphoid organs but not in the brain in fatal human infection. Am J Trop Med Hyg 1999;61:720-724. 
7. Couvelard A, Marianneau P, Bedel $C$, et al. Report of a fatal case of dengue infection with hepatitis: demonstration of dengue antigens in hepatocytes and liver apoptosis. Hum Pathol 1999;30:1106-1110.

8. Huerre MR, Lan NT, Marianneau P, et al. Liver histopathology and biological correlates in five cases of fatal dengue fever in Vietnamese children. Virchows Arch 2001;438:107-115.

9. Basílio-de-Oliveira CA, Aguiar GR, Baldanza MS, et al. Pathologic study of a fatal case of dengue-3 virus infection in Rio de Janeiro. Braz J Infect Dis 2005;9:341-347.

10. Yauch LE, Shresta S. Mouse models of dengue virus infection and disease. Antiviral Res 2008;80:87-93.

11. Paes MV, Pinhão AT, Barreto DF, et al. Liver injury and viremia in mice infected with dengue-2 virus. Virology 2005;338:236-246.

12. Nogueira RMR, Miagostovich MP, Schatzmayr HC, et al. Dengue type 2 outbreak in the south of the state of Bahia, Brazil: laboratorial and epidemiological studies. Rev Ins Med Trop SP 1995;37:507-510.

13. Erhardt W, Hebestedt A, Aschenbrenner G, et al. Comparative study with various anesthetics in mice (pentobarbitone, ketamine-xylazine, carfentanyl-etomidate. Res Exp Med 1984;184:159-169.

14. Reynolds ES. The use of lead citrate at high $\mathrm{pH}$ as an electron-opaque stain in electron microscopy. J Cell Biol 1638;17:208-212.

15. Nuovo GJ, Elton TS, Nana-Sinkam P, et al. A methodology for the combined in situ analyses of the precursor and mature forms of microRNAs and correlation with their putative targets. Nat Protoc 2009;4:107-115.

16. Huang KJ, Li SYJ, Chen SC, et al. Manifestation of thrombocytopenia in dengue-2-virus-infected mice. J Gen Virol 2000;81:2177-2182.

17. Atrasheuskaya A, Petzelbauer P, Fredeking TM, et al. Anti-TNF antibody treatment reduces mortality in experimental dengue virus infection. FEMS Immunol Med Microbiol 2003;35:33-42.

18. Boonpucknavig $S$, Vuttiviroj $O$, Boonpucknavig V. Infection of young adult mice with dengue virus type 2. Trans R Soc Trop Med Hyg 1981;75:647-653.

19. Hotta $H$, Murakami I, Miyasaki K, et al. Inoculation of dengue virus into nude mice. J Gen Viral 1981;52:71-75.

20. An J, Kimura-Kuroda J, Hirabayashi Y, et al. Development of anovel mouse model for dengue virus infection. Virology 1999;263: 70-77.

21. An J, Zhou DS, Zhang JL, et al. Dengue-specific CD8+ T cells have both protective and pathogenic roles in dengue virus infection. Immunol Lett 2004;95:167-174.

22. Shresta S, Kyle JL, Robert Beatty $P$, et al. Early activation of natural killer and $B$ cells in response to primary dengue virus infection in $A / J$ mice. Virology 2004;319:62-73.

23. Kyle JL, Beatty PR, Harris E. Dengue virus infects macrophages and dendritic cells in a mouse model of infection. J Infect Dis 2007:195:1808-1817.

24. Chaturvedi UC, Dhawan R, Rhanna M, et al. Breakdown of the bloodbrain barrier during dengue virus infection of mouse. J Gen Virol 1991;72:859-866.

25. Chen HC, Lai SY, Sung JM, et al. Lymphocyte activation and hepaticcellular infiltration in immunocompetent mice infected by dengue virus. J Med Virol 2004;73:419-431.
26. Bhamarapravati N, Tuchinda P, Boonpucknavik V. Pathology of Thailand haemorrhagic fever: a study of 100 autopsy cases. Ann Trop Med Parasitol 1967:61:500-510.

27. Burke T. Dengue haemorrhagic fever: a pathological study. Trans R Soc Trop Med Hyg 1968;62:682-692.

28. Jessie K, Fong MY, Devi S, et al. Localization of dengue virus in naturally infected human tissues, by immunohistochemistry and in situ hybridization. J Infect Dis 2004;189:1411-1418.

29. Fresh JW, Reyes V, Clarke EJ, et al. Philippine hemorrhagic fever: a clinical, laboratory, and necropsy study. J Lab Clin Med 1969;73: 451-458.

30. Quaresma JA, Barros VL, Fernandes ER, et al. Reconsideration of histopathology and ultrastructural aspects of the human liver in yellow fever. Acta Trop 2005;94:116-127.

31. Powell EE, Jonsson JR, Clouston AD. Steatosis: Co-factor in other liver diseases. Hepatology 2005;42:5-13.

32. Wong KF, Chan JK, Chan JC, et al. Dengue virus infection-associated hemophagocytic syndrome. Am J Hematol 1991;38:339-340.

33. Lu PL, Hsiao HH, Tsai JJ, et al. Dengue virus-associated hemophagocytic syndrome and dyserythropoiesis: a case report. Kaohsiung J Med Sci 2005;21:34-39.

34. Jain $D$, Singh $T$. Dengue virus related hemophagocytosis: a rare case report. Hematology 2008;13:286-288.

35. Srichaikul T, Punyagupta S, Kanchanapoom T, et al. Hemophagocytic syndrome in Dengue hemorrhagic fever with severe multiorgan complications. J Med Assoc Thai 2008;91:104-109.

36. Kuo CH, Tai DL, Chang-Chien CS, et al. Liver biochemical tests and dengue fever. Am J Trop Med Hyg 1992;47:265-270.

37. Nguyen TL, Nguyen TH, Tieu NT, et al. The impact of dengue haemorrhagic fever on liver function. Res Virol 1997;148:273-277.

38. Quaresma JA, Barros VL, Pagliari C, et al. Hepatocyte lesions and cellular immune response in yellow fever infection. Trans $\mathrm{R}$ Soc Trop Med Hyg 2007;101:161-168.

39. Souza LU, Nogueira RM, Soares LC, et al. The impact of dengue on liver function as evaluated by aminotransferase levels. Braz J Infect Dis 2007;11:407-410.

40. Marianneau $P$, Steffan AM, Royer $C$, et al. Differing infection patterns of dengue and yellow fever viruses in a human hepatoma cell line. $\mathrm{J}$ Infect Dis 1998;178:1270-1278.

41. Limonta D, Capó V, Torres $\mathrm{G}$, et al. Apoptosis in tissues from fatal dengue shock syndrome. J Clin Virol 2007;40:50-54.

42. El-Bacha T, Midlej V, Pereira da Silva AP, et al. Mitochondrial and bioenergetic dysfunction in human hepatic cells infected with dengue 2 virus. Biochim Biophys Acta 2007;72:1158-1166.

43. Mohan B, Patwari AK, Anand VK. Hepatic dysfunction in childhood dengue infection. J Trop Pediatr 2000;46:40-43.

44. Ling LM, Wilder-Smith A, Leo YS. Fulminant hepatitis in dengue haemorrhagic fever. J Clin Virol 2007;38:265-268.

45. Miagostovich MP, Ramos RG, Nicol AF, et al. Retrospective study on dengue fatal cases. Clin Neuropathol 1997;16:204-208.

46. Costa SM, Paes MV, Barreto DF, et al. Protection against dengue type vírus induced in mice immunized with a DNA plasmid encoding the non-structural 1 (NS1) gene fused to the tissue plasminogen activator signal sequence. Vaccine 2006;24:195-205. 